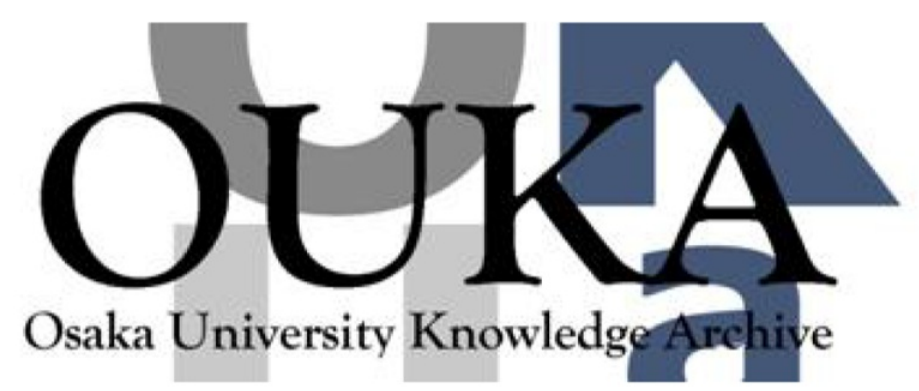

\begin{tabular}{|c|l|}
\hline Title & $\begin{array}{l}\text { Higgs inflation is still alive after the } \\
\text { results from BICEP2 }\end{array}$ \\
\hline Author(s) & Hamada, Yuta; Kawai, Hikaru; Oda, Kinya et al. \\
\hline Citation & Physical Review Letters. 112(24) p. 241301 \\
\hline Issue Date & $2014-06-16$ \\
\hline oaire:version & VoR \\
\hline URL & https://hdl. handle. net/11094/78746 \\
\hline rights & ○ 2014 American Physical Society \\
\hline Note & \\
\hline
\end{tabular}

Osaka University Knowledge Archive : OUKA

https://ir. Library. osaka-u. ac. jp/

Osaka University 


\title{
Higgs Inflation is Still Alive after the Results from BICEP2
}

\author{
Yuta Hamada, ${ }^{1, *}$ Hikaru Kawai, ${ }^{1, \dagger}$ Kin-ya Oda, ${ }^{2, \$}$ and Seong Chan Park ${ }^{3, \S}$ \\ ${ }^{1}$ Department of Physics, Kyoto University, Kyoto 606-8502, Japan \\ ${ }^{2}$ Department of Physics, Osaka University, Osaka 560-0043, Japan \\ ${ }^{3}$ Department of Physics, Sungkyunkwan University, Suwon 440-746, Korea
}

(Received 20 March 2014; published 16 June 2014)

\begin{abstract}
The observed value of the Higgs boson mass indicates that the Higgs potential becomes small and flat at the scale around $10^{17} \mathrm{GeV}$. Having this fact in mind, we reconsider the Higgs inflation scenario proposed by Bezrukov and Shaposhnikov. It turns out that the nonminimal coupling $\xi$ of the Higgs squared to the Ricci scalar can be smaller than 10 . For example, $\xi=7$ corresponds to the tensor-to-scalar ratio $r \simeq 0.2$, which is consistent with the recent observation by BICEP2.
\end{abstract}

DOI: 10.1103/PhysRevLett.112.241301

PACS numbers: $98.80 . \mathrm{Cq}, 14.80 . \mathrm{Bn}$

The observed value of the Higgs boson mass $125.9 \pm 0.4 \mathrm{GeV}[1]$ indicates that the standard model (SM) Higgs potential becomes small and flat at the scale around $10^{17} \mathrm{GeV}$; see, e.g., [2-9] for latest analyses [10]. See Fig. 1 for the Higgs potential around that scale for various values of the top quark mass [11]. We see that by tuning the top quark mass, we can make the first derivative at the inflection point arbitrarily small as shown by the blue (center) line. Note that the required tuning of the top quark mass is rather strict. The values of $M_{t}$ are given to show the amount of tuning and should not be taken literally [17]. There are several arguments that this tuning is required by a principle such as the multiple point principle [20-22], the maximum entropy principle [23,24], the classical conformality [25-32], and the asymptotic safety [33].

It is known that this inflection point cannot be used to achieve a successful inflation [34-36]. Slow-roll condition $\left|\eta_{V}\right| \lesssim 1$ restricts the field value to be very close to the inflection point. To earn a sufficient $e$ folding $N_{*} \simeq 60$ within this range of $\varphi_{*}$, the first derivative at the inflection point must be very small, and hence cannot yield the right amount of the amplitude $A_{s} \propto V_{*} / \epsilon_{V}$ at $\varphi_{*}$.

In Ref. [35], we have discussed a possibility that a new physics, such as string theory, modifies the Higgs potential above the scale $\Lambda \sim 10^{17} \mathrm{GeV}$. In this Letter, we pursue another possibility that the nonminimal coupling of the Higgs squared to the Ricci scalar, $\xi \varphi^{2} \mathcal{R}$, leads to a successful inflection point inflation.

The main differences from the ordinary Higgs inflation scenario [39-43] are the following two points [44]: (i) The $e$ folding is earned in passing the inflection point, and hence the relation $\epsilon_{V} \sim 1 / N_{*}^{2}$ no longer holds. Therefore, the scalar-to-tensor ratio $r=16 \epsilon_{V}$ can be sizable to match the recent BICEP2 result [49]:

$$
r=0.2_{-0.05}^{+0.07}
$$

at the $68 \%$ C.L. (ii) $\xi$ can be smaller than 10 , since the Higgs quartic coupling $\lambda$ is small at $\varphi_{*}$, due to the tuning mentioned above.

We start from the same Lagrangian as the ordinary Higgs inflation $[39,41,42,50]$. The potential in the Einstein frame can be obtained from the effective potential

$$
V(\varphi)=\frac{\lambda(\varphi)}{4} \varphi^{4}
$$

in the flat space, by setting $\varphi=\varphi_{h}$ with

$$
\varphi_{h}:=\frac{h}{\sqrt{1+\xi h^{2} / M_{P}^{2}}},
$$

where $h$ is the Higgs field in the Jordan frame [53].

The running coupling $\lambda(\mu)$ has a minimum at $\mu_{\min } \sim 10^{17-18} \mathrm{GeV}$, depending on the Higgs boson mass [2-9,54]. Around the minimum, $\lambda(\mu)$ can be expanded as

$$
\begin{aligned}
\lambda(\mu)= & \lambda_{\min }+\frac{\beta_{2}}{\left(16 \pi^{2}\right)^{2}}\left(\ln \frac{\mu}{\mu_{\min }}\right)^{2} \\
& +\frac{\beta_{3}}{\left(16 \pi^{2}\right)^{3}}\left(\ln \frac{\mu}{\mu_{\min }}\right)^{3}+\cdots,
\end{aligned}
$$

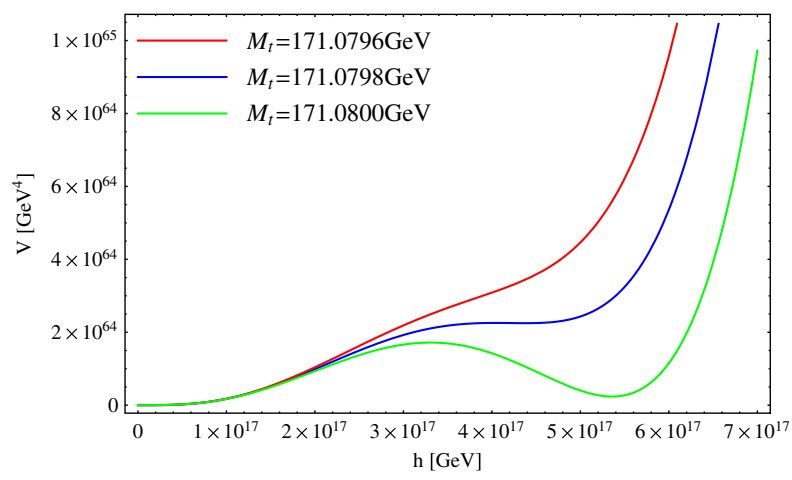

FIG. 1 (color online). Standard model Higgs potential for the Higgs boson mass $125.6 \mathrm{GeV}$. 

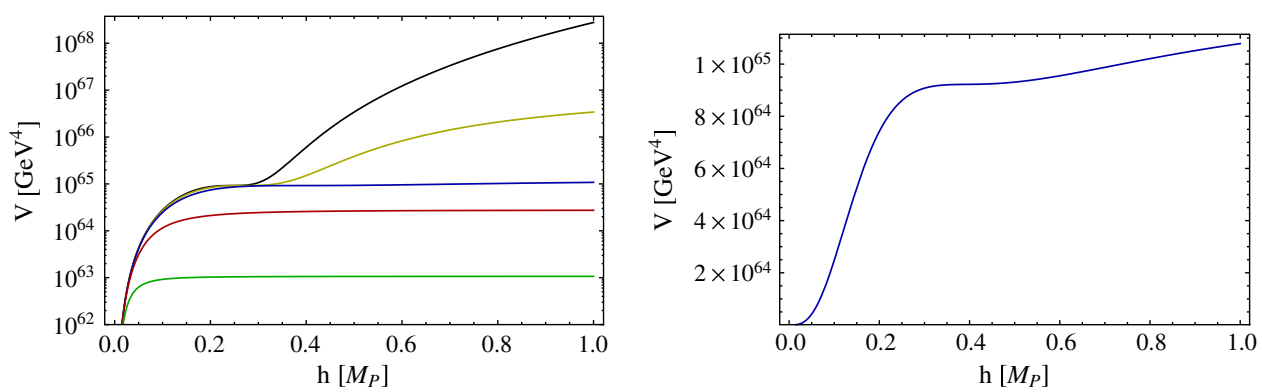

FIG. 2 (color online). Left: Inflaton potential for $\xi=0,3,10,100$, and 1000 from above to below in a log-linear plot. Right: the same for $\xi=10$ in a linear-linear plot.

where $\beta_{2} \simeq 0.6$ in the SM [35]. The terms proportional to $\beta_{3}$ and higher are small in the region of our interest, and we will neglect them hereafter. The value of $\lambda_{\text {min }}$ depends on the top quark mass, and we can set it arbitrarily small by tuning the top quark mass within the current experimental bound.

For the potential $V(\varphi)$ to be monotonically increasing around the inflection point, it is necessary and sufficient that

$$
\lambda_{\min } \geq \lambda_{c}:=\frac{\beta_{2}}{\left(64 \pi^{2}\right)^{2}} \sim 10^{-6}
$$

The equality holds when the potential has a plateau. That is, when we put $\lambda_{\min }=\lambda_{c}$, the point $\varphi_{\text {inflection }}=e^{-1 / 4} \mu_{\min } \simeq$ $0.8 \mu_{\min }$ becomes a saddle point with vanishing first and second derivatives [55].

We set the value of $\lambda_{\min }$ slightly larger than $\lambda_{c}$ to realize an inflection point inflation, while keeping the potential above $\varphi_{\text {inflection }}$ sufficiently small by the introduction of $\xi$ in order to evade the problem described above. The three cases $\lambda>\lambda_{c}, \lambda=\lambda_{c}$, and $\lambda<\lambda_{c}$ correspond to the red (upper), blue (middle), and green (lower) curves in Fig. 1, respectively. An important point here is that the value of $\varphi_{h}$ in Eq. (3) is saturated to $M_{P} / \sqrt{\xi}$ for large values of $h$ $\left(\gg M_{P} / \sqrt{\xi}\right.$ ), and therefore the potential does not grow rapidly. In order for this saturation to work to avoid too large $\eta_{V}$, we need $\varphi_{\text {inflection }} \sim M_{P} / \sqrt{\xi}$, that is, $\xi \sim M_{P}^{2} / \mu_{\min }^{2}$.

As concrete examples, we show our results for several benchmark points with the parameter choice $\xi=0,3,10$, 100 , and 1000 with $\lambda_{\min }=1.01 \lambda_{c}, \beta_{2}=0.6$, and $\mu_{\min }=$ $M_{P} / \sqrt{10}$ in the left panel in Fig. 2; the same figure is drawn in a linear plot for $\xi=10$ in the right panel.

To fit the cosmological data, we can, e.g., take $h_{*}=0.896 M_{P}, \lambda_{\min }=1.01 \lambda_{c}, \mu_{\min }=0.37 M_{P}, \xi=7$ to get $\quad r=16 \epsilon_{V}\left(h_{*}\right)=0.19, \quad N_{*}=58, \quad V\left(\varphi_{h_{*}}\right) / \epsilon_{V}\left(h_{*}\right)=$ $5.0 \times 10^{-7}$ and $n_{s}\left(h_{*}\right)=0.955$, where

$$
\begin{aligned}
\epsilon_{V} & =\frac{M_{P}^{2}}{2 V\left(\varphi_{h}\right)^{2}}\left(\frac{d h}{d \chi} \frac{d V\left(\varphi_{h}\right)}{d h}\right)^{2}, \\
\eta_{V} & =\frac{M_{P}^{2}}{V\left(\varphi_{h}\right)} \frac{d h}{d \chi} \frac{d}{d h}\left(\frac{d h}{d \chi} \frac{d V\left(\varphi_{h}\right)}{d h}\right),
\end{aligned}
$$

with

$$
\frac{d \chi}{d h}=\frac{\sqrt{1+\xi(1+6 \xi) h^{2} / M_{P}^{2}}}{1+\xi h^{2} / M_{P}^{2}}
$$

For the same parameters, the Einstein-frame time evolution of the Higgs field $h$ is plotted in Fig. 3. We see that substantial time is spent around the inflection point.

Once the tensor-to-scalar ratio is fixed to be $r \simeq 0.2$, the slow-roll parameter becomes $\epsilon_{V}\left(h_{*}\right) \simeq 0.013$, and the amplitude $A_{s} \propto V\left(\varphi_{h_{*}}\right) / \epsilon_{V}\left(h_{*}\right)$ fixes the potential height $V\left(\varphi_{h_{*}}\right)^{1 / 4} \simeq 2 \times 10^{16} \mathrm{GeV}$. The potential height is determined in our case to be $V\left(\varphi_{h_{*}}\right) \simeq \lambda\left(\varphi_{h_{*}}\right) M_{P}^{4} / \xi^{2}$, which is the same as the Higgs inflation. The difference is the value of $\lambda\left(\varphi_{h_{*}}\right) \simeq \lambda_{\min } \simeq \lambda_{c} \sim 10^{-6}$ that allows us to take $\xi \lesssim 10$.

In this Letter, we have matched the renormalization scale in the Einstein frame, as in Eq. (3). If we instead match it in the Jordan frame [56], i.e., if we set $\varphi=h$ in Eq. (2), we obtain the chaotic inflation at $h \gg M_{P} / \sqrt{\xi}$. In this region, the canonically normalized field is $\hat{\chi} \simeq$ $\sqrt{6} M_{P} \ln \left(h M_{P} / \sqrt{\xi} \mu_{\min }^{2}\right)$ in the Einstein frame. The potential for $\hat{\chi}$ becomes quadratic:

$$
V \simeq \frac{\lambda_{\min } M_{P}^{4}}{4 \xi^{2}}+\frac{1}{2} \frac{\beta_{2} M_{P}^{2}}{48 \xi^{2}\left(16 \pi^{2}\right)^{2}} \hat{\chi}^{2} .
$$

We see that by taking $\xi \sim 100$, we get the right amount of the inflaton mass $\sim 10^{13} \mathrm{GeV}$.

Finally, we comment on the unitarity issue in the Higgs inflation due to the large nonminimal coupling, which

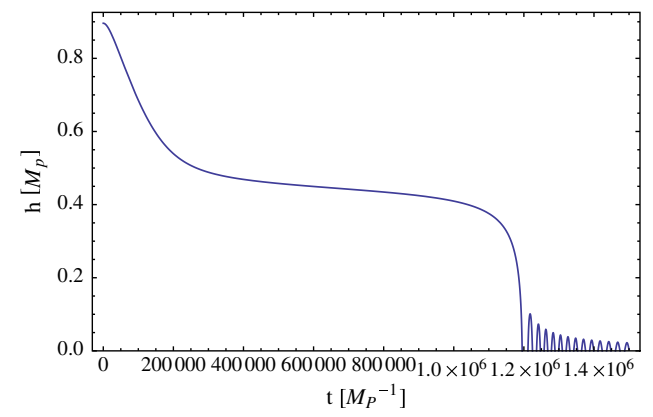

FIG. 3 (color online). $h$ vs $t$ in the Einstein frame in Planck units. 
requires a new physics above the scale $\Lambda \sim M_{P} / \xi$ in order to cure the scattering being strongly coupled on the electroweak vacuum $\left(\varphi \ll M_{P} / \sqrt{\xi}\right)$ [59-64]. It is an implicit assumption of the Higgs inflation that such an extension does not affect the result qualitatively, that is, the Wilson coefficients of the higher order terms are sufficiently smaller than $1 / \xi$. Note that our $\xi$ is greatly reduced from the value $\xi \sim 10^{5}$ in the ordinary scenario.

K. O. and S. C. P. thank Takuya Kakuda and Jinsu Kim for useful discussions. We thank Yukinari Sumino for the useful comments. The work of Y. H. is partly supported by a Grant-in-Aid from the Japan Society for the Promotion of Science (JSPS) Fellows No. 25.1107. S. C. P. is supported by the Basic Science Research Program through the National Research Foundation of Korea funded by the Ministry of Education, Science and Technology (2011-0010294) and (2011-0029758) and (NRF2013R1A1A2064120). The work of K. O. is in part supported by the Grant-in-Aid for Scientific Research Grants No. 23104009, No. 20244028, and No. 23740192.

Note added.-After submission of this Letter, there appeared an article treating a similar subject [65], which is consistent with our result [66].

*hamada@gauge.scphys.kyoto-u.ac.jp

†hkawai@gauge.scphys.kyoto-u.ac.jp

*odakin@phys.sci.osaka-u.ac.jp

\$s.park@skku.edu

1

[1] J. Beringer et al. (Particle Data Group), Phys. Rev. D 86, 010001 (2012), and 2013 partial update for the 2014 edition.

[2] M. Holthausen, K. S. Lim, and M. Lindner, J. High Energy Phys. 02 (2012) 037.

[3] F. Bezrukov, M. Y. Kalmykov, B. A. Kniehl, and M. Shaposhnikov, J. High Energy Phys. 10 (2012) 140.

[4] G. Degrassi, S. Vita, J. Elias-Miró, J. R. Espinosa, G. F. Giudice, G. Isidori, and A. Strumia, J. High Energy Phys. 08 (2012) 098.

[5] S. Alekhin, A. Djouadi, and S. Moch, Phys. Lett. B 716, 214 (2012).

[6] I. Masina, Phys. Rev. D 87, 053001 (2013).

[7] Y. Hamada, H. Kawai, and K.-y. Oda, Phys. Rev. D 87, 053009 (2013).

[8] F. Jegerlehner, arXiv:1304.7813.

[9] D. Buttazzo, G. Degrassi, P. P. Giardino, G. F. Giudice, F. Sala, A. Salvio, and A. Strumia, J. High Energy Phys. 12 (2013) 089.

[10] It is an intriguing fact that the bare Higgs mass also becomes small at the same scale [7,11,12]; see also Refs. [13-15]. The running Higgs boson mass after the subtraction of the quadratic divergence is considered, e.g., in Ref. [16].

[11] Y. Hamada, H. Kawai, and K.-y. Oda, arXiv:1305.7055.

[12] I. Masina and M. Quiros, Phys. Rev. D 88, 093003 (2013).

[13] M. Al-sarhi, I. Jack, and D. Jones, Z. Phys. C 55, 283 (1992).

[14] D. Jones, Phys. Rev. D 88, 098301 (2013).
[15] N. Haba, K. Kaneta, and R. Takahashi, J. High Energy Phys. 04 (2014) 029.

[16] L. Bian, Phys. Rev. D 88, 056022 (2013).

[17] The latest combined result for the top quark mass is $173.34 \pm 0.76 \mathrm{GeV}$ [18]. Note that there can be a discrepancy between the pole mass $M_{t}$ and the one measured at the hadron colliders; see, e.g., Refs. [5,11]. The latter is obtained as an invariant mass of the color singlet final states, whereas the former is a pole of a colored quark. At the hadron colliders, the observed $t \bar{t}$ pair is dominantly color octet, and there may be discrepancy of order $1-2 \mathrm{GeV}$ in drawing extra lines to make the singlet final states. We thank Yukinari Sumino on this point. See also Ref. [19].

[18] D0, ATLAS, CDF, and CMS Collaborations, arXiv:1403.4427.

[19] T. Horiguchi, A. Ishikawa, T. Suehara, K. Fujii, Y. Sumino, Y. Kiyo, and H. Yamamoto, arXiv:1310.0563.

[20] C. Froggatt and H. B. Nielsen, Phys. Lett. B 368, 96 (1996).

[21] C. D. Froggatt, H. B. Nielsen, and Y. Takanishi, Phys. Rev. D 64, 113014 (2001).

[22] H. B. Nielsen, arXiv:1212.5716 [Proceedings to the 15 th Bled Workshop (to be published)], p. 94.

[23] H. Kawai and T. Okada, Prog. Theor. Phys. 127, 689 (2012).

[24] H. Kawai, Int. J. Mod. Phys. A 28, 1340001 (2013).

[25] K. A. Meissner and H. Nicolai, Phys. Lett. B 648, 312 (2007).

[26] K. A. Meissner and H. Nicolai, Phys. Lett. B 660, 260 (2008).

[27] S. Iso, N. Okada, and Y. Orikasa, Phys. Lett. B 676, 81 (2009).

[28] S. Iso, N. Okada, and Y. Orikasa, Phys. Rev. D 80, 115007 (2009).

[29] H. Aoki and S. Iso, Phys. Rev. D 86, 013001 (2012).

[30] S. Iso and Y. Orikasa, Prog. Theor. Exp. Phys. 023B08 (2013).

[31] M. Hashimoto, S. Iso, and Y. Orikasa, Phys. Rev. D 89, 016019 (2014).

[32] M. Hashimoto, S. Iso, and Y. Orikasa, Phys. Rev. D 89, 056010 (2014).

[33] M. Shaposhnikov and C. Wetterich, Phys. Lett. B 683, 196 (2010).

[34] G. Isidori, V. S. Rychkov, A. Strumia, and N. Tetradis, Phys. Rev. D 77, 025034 (2008).

[35] Y. Hamada, H. Kawai, and K.-y. Oda, Prog. Theor. Exp. Phys. 023B02 (2014).

[36] See, e.g., Refs. [37,38] for attempts of the inflection point inflation.

[37] R. Allahverdi, K. Enqvist, J. Garcia-Bellido, and A. Mazumdar, Phys. Rev. Lett. 97, 191304 (2006).

[38] T. Imai and K. I. Izawa, arXiv:1305.5307.

[39] F. L. Bezrukov and M. Shaposhnikov, Phys. Lett. B 659 , 703 (2008).

[40] S. C. Park and S. Yamaguchi, J. Cosmol. Astropart. Phys. 08 (2008) 009.

[41] F. Bezrukov and M. Shaposhnikov, J. High Energy Phys. 07 (2009) 089.

[42] F. Bezrukov, A. Magnin, M. Shaposhnikov, and S. Sibiryakov, J. High Energy Phys. 01 (2011) 016.

[43] A. Salvio, Phys. Lett. B 727, 234 (2013).

[44] For the other attempts, see Refs. [45-48].

[45] K. Kamada, T. Kobayashi, M. Yamaguchi, and J. Yokoyama, Phys. Rev. D 83, 083515 (2011). 
[46] K. Kamada, T. Kobayashi, T. Takahashi, M. Yamaguchi, and J. Yokoyama, Phys. Rev. D 86, 023504 (2012).

[47] K. Kamada, T. Kobayashi, T. Kunimitsu, M. Yamaguchi, and J. Yokoyama, Phys. Rev. D 88, 123518 (2013).

[48] K. Nakayama and F. Takahashi, arXiv:1403.4132.

[49] P. A. R. Ade et al. (BICEP2 Collaboration), arXiv:1403.3985.

[50] Here we are treating the Higgs inflation as a single-field model, as in Refs. [39,41,42]. Even when the NambuGoldstone bosons are explicitly included, the multifield version of Higgs inflation rapidly evolves as an effectively single-field model due to the dynamics of the system's evolution $[51,52]$, rather than by tacitly assuming unitary gauge. The analysis in Ref. [51] did not incorporate quantum corrections as the present Letter does, but, nonetheless, based on Ref. [51] as well as Ref. [52], it should be clear that the single-field (dynamical) attractor behavior holds whenever the nonminimal coupling is sufficiently large.

[51] R. N. Greenwood, D. I. Kaiser, and E. I. Sfakianakis, Phys. Rev. D 87, 064021 (2013).

[52] D. I. Kaiser and E. I. Sfakianakis, Phys. Rev. Lett. 112, 011302 (2014).

[53] This choice corresponds to the prescription I in Ref. [41], which minimizes the one-loop logarithmic correction to the effective potential in the Einstein frame.

[54] The Higgs quartic coupling grows above the minimum due to the contribution of the growing $U(1)_{Y}$ coupling. Qualitatively, the position and height of the minimum depend on the Higgs boson and top quark masses, respectively.

[55] There appears another inflection point at $e^{-11 / 12} \mu_{\min } \simeq$ $0.4 \mu_{\min }$ too.
[56] It is argued in Ref. [57] that these two choices correspond to different theories. The choice $\varphi=h$ corresponds to the prescription II in Ref. [41], which minimizes the one-loop logarithmic correction in the Jordan frame. We note, however, that there is a criticism on this choice [58]. Whether calculations involving quantum corrections are or are not frame dependent is a point of ongoing research, and we leave it open for further discussion.

[57] F. Bezrukov, Classical Quantum Gravity 30, 214001 (2013).

[58] D. P. George, S. Mooij, and M. Postma, J. Cosmol. Astropart. Phys. 02 (2014) 024.

[59] C. P. Burgess, H. M. Lee, and M. Trott, J. High Energy Phys. 09 (2009) 103.

[60] J. L. F. Barbon and J. R. Espinosa, Phys. Rev. D 79, 081302 (2009).

[61] C. P. Burgess, H. M. Lee, and M. Trott, J. High Energy Phys. 07 (2010) 007.

[62] G. F. Giudice and H. M. Lee, Phys. Lett. B 694, 294 (2011).

[63] C. P. Burgess, S. P. Patil, and M. Trott, arXiv:1402.1476.

[64] We note that the cutoff scale depends on the background field value $\varphi$, and for $\varphi \gtrsim M_{P} / \sqrt{\xi}$, it becomes $\Lambda \sim$ $\sqrt{M_{P}^{2}+\xi \varphi^{2}}$ [42]. That is, inflation takes place below the cutoff scale.

[65] J. L. Cook, L. M. Krauss, A. J. Long, and S. Sabharwal, Phys. Rev. D 89, 103525 (2014).

[66] In Ref. [65], the result of Ref. [67] in the prescription II is used.

[67] K. Allison, J. High Energy Phys. 02 (2014) 040. 